\title{
Large Magnetoresistance Ratio in Ferromagnetic Single-Electron Transistors in the Strong Tunneling Regime
}

\author{
X.H. Wang \\ Department of Theoretical Physics, Lund University, Helgonavägen 5, S-223 62 Lund, Sweden \\ Arne Brataas* \\ Department of Applied Physics and Delft Institute of Microelectronics and Submicrontechnology (DIMES), Delft University of \\ Technology, Lorentzweg 1, 2628 CJ Delft, The Netherlands
}

(Received 2 August 1999)

\begin{abstract}
We study transport through a ferromagnetic single-electron transistor. The resistance is represented as a path integral, so that systems where the tunnel resistances are smaller than the quantum resistance can be investigated. Beyond the low-order sequential tunneling and cotunneling regimes, a large magnetoresistance ratio at sufficiently low temperatures is found. In the opposite limit, when the thermal energy is larger than the charging energy, the magnetoresistance ratio is only slightly enhanced.
\end{abstract}

PACS numbers: 75.70.Pa, 73.23.Hk, 73.40.Gk

Electron tunneling between two ferromagnetic metallic leads separated by an insulating layer has been studied since the 1970s [1]. The novel feature of these structures is that the conductance depends on the relative orientation of the magnetizations in the leads. By tuning the external magnetic field, the magnetization of a ferromagnetic metal can be controlled, and hence the conductance of the device changes. The influence of the applied magnetic field on the transport properties is characterized by the tunnel magnetoresistance ratio (TMR):

$$
\gamma=\left(R_{\mathrm{AP}}-R_{\mathrm{P}}\right) / R_{\mathrm{P}}
$$

where $R_{\mathrm{P}}\left(R_{\mathrm{AP}}\right)$ is the resistance when the magnetizations in the leads are parallel (antiparallel). Devices with large TMR can be used in future magnetoresistive sensors or magnetic random access memories [2].

In the past years, reproducible measurements of TMR have been reported [2-6] in structures with ultrasmall tunnel junctions. When the capacitances of the tunnel junctions are so small that the charging energy is larger than the thermal energy, the current flow is blocked when the bias voltage is smaller than a critical value determined by the junction capacitances. Such a Coulomb blockade of electron tunneling through insulating barriers has been widely investigated when the leads are nonmagnetic [710]. The single-electron transistor consists of a metallic island connected to two metallic leads via tunnel junctions and coupled to a gate-voltage $U_{g}$ via a capacitor $C_{g}$. The lowest-order perturbation in terms of the dimensionless tunnel conductance $\alpha=R_{K} / R_{T}$, where $R_{K} \equiv h / e^{2}$ is the quantum resistance and $R_{T}$ is the tunnel resistance, predicts that when the thermal energy $k_{B} T$ is much smaller than the charging energy $E_{c}=e^{2} / 2 C$, the conductance of the single-electron transistor (SET) vanishes for $C_{g} U_{g} / e=n$, and the conductance attains a maximum for $C_{g} U_{g} / e=(n+1 / 2)$, where $n$ is an integral number [8]. When the tunnel resistances $R_{T}$ are not much smaller than the quantum resistance, the Coulomb blockade of electron tunneling is softened due to cotunneling and higher-order tunneling processes [7].

In order to describe ferromagnetic single-electron transistors (FSET's), the spin dependence of the density of states and the transition matrix elements must be taken into account. Theoretical works on this subject based on the lowest-order sequential tunneling processes have recently been carried out $[11-, 13]$. By including the cotunneling processes in the Coulomb blockade regime, it has been shown $[14,15]$ that the TMR is larger in such ultrasmall structures than in macroscopic devices, where the charging energy is negligible. When the junction resistances are smaller than the quantum resistance, the calculations based on sequential tunneling or cotunneling become inaccurate because higher-order tunneling processes determine the transport properties. It is so far unclear if the TMR of the FSET is further enhanced by the higher-order tunneling processes. This question cannot be addressed from the results of the sequential tunneling or cotunneling formulations. We will employ a nonperturbative approach to clarify this issue and show that indeed there is a further large enhancement of the TMR in the strong tunneling regime as compared with the weak tunneling case where the junction resistances are larger than the quantum resistance. This enhancement of the TMR is much larger than what calculations based on the cotunneling processes predict.

We consider the situation when the two leads are made of the same material and have the same magnetization directions, and denote the resistance of the FSET as $R_{\eta}$, where $\eta=\mathrm{P}(\eta=\mathrm{AP})$ for the case that the magnetization of the central grain is parallel (antiparallel) to the magnetization in the leads $[4,11,12-14]$. The magnetoresistance ratio is defined by Eq. (1). In order to calculate the resistance of the FSET for arbitrary tunneling resistances the tunneling Hamiltonian must be treated in a nonperturbative manner. This can be realized via the 
path integral approach [9]. We use the standard singleelectron tunneling Hamiltonian with spin-dependent density of states and tunneling matrix elements $[11,14]$ and limit our discussions to the linear response regime.

At sufficiently high temperatures $\left(k_{B} T \gg E_{c}\right)$ the system is in the classical regime, the Coulomb charging effects are negligible, and the conductance of the system can be readily found. The dimensionless conductance of the FSET for the parallel magnetic alignments is $\alpha_{\mathrm{P}}^{\mathrm{cl}}=$ $\alpha_{\mathrm{M}}\left[1+(1-P)\left(1-P^{\prime}\right) /(1+P)\left(1+P^{\prime}\right)\right]$, where $\alpha_{\mathrm{M}}$ is the dimensionless majority-band conductance of the FSET with parallel magnetic alignments, and the spin polarizations of the leads and island are denoted by $P$ and $P^{\prime}$, respectively. Similarly, the dimensionless conductance of the FSET for the antiparallel magnetic alignments is $\alpha_{\mathrm{AP}}^{\mathrm{cl}}=\alpha_{\mathrm{M}}\left[(1-P) /(1+P)+\left(1-P^{\prime}\right) /\left(1+P^{\prime}\right)\right]$. The classical (high-temperature) magnetoresistance ratio is

$$
\gamma^{\mathrm{cl}} \equiv \frac{R_{\mathrm{AP}}^{\mathrm{cl}}-R_{\mathrm{P}}^{\mathrm{cl}}}{R_{\mathrm{P}}^{\mathrm{cl}}}=\frac{2 P P^{\prime}}{1-P P^{\prime}} .
$$

At lower temperatures the Coulomb charging effects must be taken into account. In this case, the resistance of the FSET can be calculated via Kubo's formula [10],

$$
\frac{R_{\eta}^{\mathrm{cl}}}{R_{\eta}}=4 \pi \lim _{\omega \rightarrow 0} \omega^{-1} \Im m\left\{\lim _{i \omega_{l} \rightarrow \omega+i \delta} \int_{0}^{\beta} d \tau e^{i \omega_{l} \tau} r(\tau)\right\},
$$

where the Matsubara frequencies are $\omega_{l}=2 \pi l / \beta(l$ is an integral number) and

$$
\begin{aligned}
r(\tau)= & Z_{\eta}^{-1} \sum_{k=-\infty}^{\infty} e^{2 \pi i k n_{\mathrm{ex}}} \\
& \times \int_{b_{k}} D \varphi e^{-S_{\eta}[\varphi]} \chi(\tau) \cos [\varphi(\tau)-\varphi(0)],
\end{aligned}
$$

where the damping kernel $\chi(\tau)$ is an even function with a period $\beta$ and Fourier components $\chi\left(\omega_{l}\right)=-\left|\omega_{l}\right| / 4 \pi$ and $n_{\mathrm{ex}}=C_{g} V_{g} / e$ is controlled by the gate voltage. In Eq. (4) the boundary condition for the path integral is $b_{k} \rightarrow \varphi(\beta)=\varphi(0)+2 \pi k$ and $Z_{\eta}$ is the partition function of the unbiased device, namely a single-electron box (SEB) [16]. $\quad Z_{\eta}$ is readily represented as a path integral, $[9,10]$,

$$
Z_{\eta}=\sum_{k=-\infty}^{\infty} e^{2 \pi i k n_{\mathrm{ex}}} \int_{b_{k}} D \varphi e^{-S_{\eta}[\varphi]} .
$$

The action is given by

$$
\begin{aligned}
S_{\eta}[\varphi]= & \int_{0}^{\beta} d \tau \frac{\dot{\varphi}^{2}(\tau)}{4 E_{c}} \\
& -\alpha_{\eta}^{\mathrm{cl}} \int_{0}^{\beta} d \tau \int_{0}^{\beta} d \tau^{\prime} \chi\left(\tau-\tau^{\prime}\right) \\
& \times \cos \left[\varphi(\tau)-\varphi\left(\tau^{\prime}\right)\right],
\end{aligned}
$$

which depends on the relative orientations of the magnetizations in the central grain and the leads through the second term in Eq. (6) describing the contribution of the tunnel junctions. If this term is neglected, the charging energy determines the action, and the sequential tunneling processes are recovered. If one expands the partition function (5) in terms of the dimensionless conductance, a Taylor series is obtained containing sequential tunneling, cotunneling, and higher-order contributions. However, in practice, it is quite tedious to evaluate the path integral term by term in this way. Instead, we will evaluate the dc current nonperturbatively.

Note that the current-current correlation functions of the SET contain both sine and cosine terms in the phase differences, as described in Ref. [10]. However, using current conservation through the first and the second junction it can be seen that the contribution from the sine term to the conductance is proportional to the contribution from the cosine term and we obtain the simplified expression (4).

Let us first discuss the most interesting case when the Coulomb charging energy is much larger than the thermal energy and there is a strong influence of the Coulomb charging on the current. In this case the conductance of the FSET can be varied by tuning the gate voltage $[8,10]$. We use Monte Carlo simulations to compute $r(\tau)(4)$, and then calculate the conductances via Kubo's formula (3). Since in the Coulomb blockade regime, the TMR for integer $n_{\mathrm{ex}}$ is at a maximum $[4,14]$, we will concentrate on this case to find the largest TMR and we set $n_{\mathrm{ex}}=0$. The problem is reduced to the evaluation of expectation values, which we will treat by Monte Carlo simulations [17].

The computations have been carried out using the standard Metropolis algorithm. The convergence of the simulations has been checked by increasing the number of Trotter indices, which are the numbers of slices in the imaginary time interval $[0, \beta]$ and are found to scale linear in $\beta$. Therefore at low temperatures where $\beta$ is large the simulations are time consuming. Samplings have been done every five passes, and the program has been run for a sufficiently long time to equilibrate the system before sampling. In order to guarantee a high precision of the Monte Carlo simulations, the number of samplings has been determined so that the result with doubled sampling number $2 N$ is the same as the result with sampling number $N$. The typical number of samplings is found to be of the order of $10^{6}$ for the parameters described below. This procedure is necessary because we calculate the Fourier components from the Monte Carlo data and then carry out the analytical continuation (3) using the Páde approximate to get the conductance of the FSET $[18,19]$.

When the tunnel resistances are close to the quantum resistance, the cotunneling result of the conductance of the SET $[8,14,20]$ is recovered. In order to find out if higherorder tunneling processes will enhance the TMR, we show the result for the conductance of a typical value in the strong tunneling case, namely $\alpha_{\mathrm{M}}=10$. We consider $\mathrm{Ni}$ leads with spin polarization $P=0.23$, and Co island with $P^{\prime}=0.35$, as in the experiment by Ono et al. [4]. For this 
device, the prediction of the calculations of the TMR in the classical regime (high temperature) yields a value of $17.5 \%$ and in the Coulomb blockade regime the cotunneling formalism gives a value of $38 \%$ [14]. The computed results of the TMR as a function of $E_{c} / k_{B} T$ are shown in Fig. 1. In the low-temperature quantum transport regime, the TMR is denoted by $\gamma^{\text {qu }}$. We see that the TMR is strongly enhanced by lowering the temperature and reaches the cotunneling result [14] when $E_{c} / k_{B} T \simeq 15$. In the case of cotunneling, the TMR saturates at this temperature and remains constant at lower temperatures. We would like to emphasize that in our case by including higherorder tunneling processes a further large enhancement of the TMR occurs at even lower temperatures, e.g., when $E_{c} / k_{B} T \simeq 40$ the TMR is enhanced to $90 \%$, which is a much larger value than in the cotunneling regime. At even lower temperatures a further enhancement is expected. The fact that there is a large enhancement of the TMR in the strong tunneling regime can be understood as a consequence of higher-order tunneling. In the cotunneling regime the conductance of the device is proportional to the square of the tunnel conductance which roughly doubles the TMR with respect to the classical value. In our case with $\alpha_{\mathrm{M}}=10$ the conductance increases even faster than the square of the tunnel conductance and hence the TMR is further enhanced. The numerical results of the TMR in the low-temperature quantum regime can roughly be fitted by

$$
\gamma^{\mathrm{qu}}=\gamma^{\mathrm{cl}}+\mu^{\mathrm{qu}}\left(1+\gamma^{\mathrm{cl}}\right)\left(E_{c} / k_{B} T\right)^{2},
$$

where we choose $\mu^{\mathrm{qu}}=0.0006$ so that the fitting curve is close to most of the data. Note that although $\mu^{\text {qu }}$ is quite small, the second term on the right hand side of (7) can be as large as unity when the temperatures are sufficiently

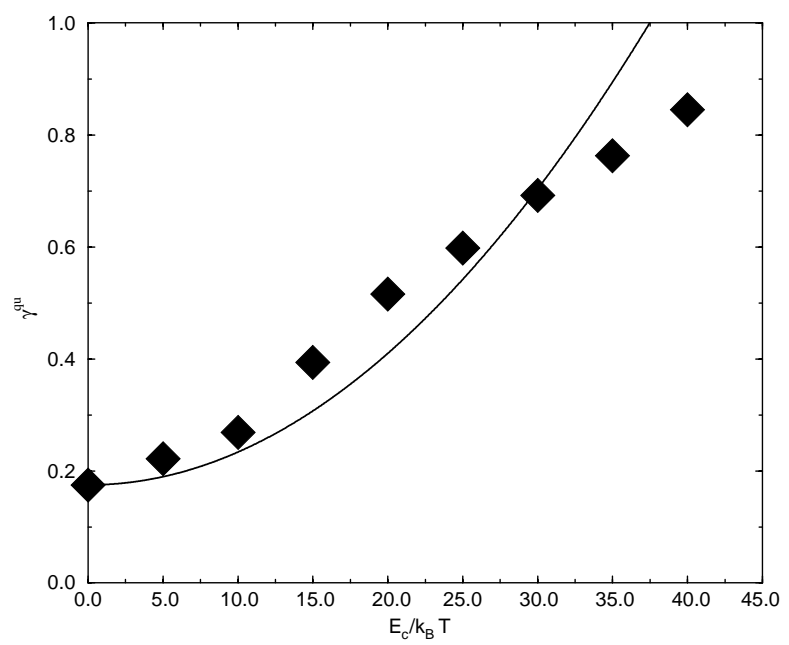

FIG. 1. The tunnel magnetoresistance ratio (TMR) as a function of the dimensionless charging energy in the strong tunneling regime for $\alpha_{\mathrm{M}}=10$. The diamonds are Monte Carlo data with errors smaller than the sizes, and the solid line is calculated via Eq. (7) for $\mu^{\text {qu }}=0.0006$. low leading to a large enhancement of the TMR. We have thus shown that there is a large enhancement of the TMR in the strong tunneling regime at low temperatures, which is well beyond the results from the sequential tunneling and cotunneling formalisms.

Let us now consider the semiclassical regime when the thermal energy $k_{B} T$ is larger than the Coulomb charging energy $E_{c}$. The fluctuation amplitude of the phase variable can then be estimated from the kinetic part of the action giving a value of the order of $\left[E_{c} / k_{B} T\right]^{1 / 2}$, and the dominating part of the action is Gaussian with some corrections from the nonquadratic parts of the tunneling term in the total action. By expanding the cosine function in the action Eq. (6) and in Eq. (4), the resistance of the FSET to the second order in $E_{c} / k_{B} T$ is

$$
\frac{R_{\eta}^{\mathrm{se}}}{R_{\eta}^{\mathrm{cl}}}=1+\frac{E_{c}}{3 k_{B} T}+\left(0.04-0.02 \alpha_{\eta}^{\mathrm{cl}}\right)\left(\frac{E_{c}}{k_{B} T}\right)^{2} .
$$

The calculation leading to the above equation is very similar to the one of the nonmagnetic SET carried out in Ref. [10]. The two first terms in Eq. (8) have also been obtained in Ref. [14]. The third term could also have been derived from the formalism used in Ref. [14], but the last term is due to higher-order tunneling. It is only this term that gives a temperature-dependent contribution to the TMR at high temperatures. The TMR in the semiclassical regime is

$$
\gamma^{\mathrm{se}}=\gamma^{\mathrm{cl}}+\mu^{\mathrm{se}}\left(1+\gamma^{\mathrm{cl}}\right)\left(E_{c} / k_{B} T\right)^{2},
$$

where

$$
\mu^{\mathrm{se}}=0.04 \alpha_{\mathrm{M}}\left(1+P P^{\prime}\right) /\left[(1+P)\left(1+P^{\prime}\right)\right] .
$$

For $P=0.23$ and $P^{\prime}=0.35$, we find $\mu^{\mathrm{se}} \approx 0.013 \alpha_{\mathrm{M}}$ and the TMR for intermediate Coulomb charging energy in the strong tunneling regime, e.g., for $E_{c}=k_{B} T / 4$ and $\alpha_{\mathrm{M}}=10$, increases by less than $1 \%$ giving a TMR much smaller than the low-temperature cotunneling result of $38 \%$. The above analysis shows that when the thermal energy is dominating, the Coulomb charging effect does not drastically change the TMR, and the role of the higher-order tunneling processes is not very important. In order to make the TMR significantly different from the corresponding values of a macroscopic device, one has to minimize the junction capacitances so that the devices work in the low-temperature regime.

It is interesting to note that the enhancement of the TMR in the low-temperature quantum regime (7) has a similar temperature dependence as the enhancement of the TMR in the high-temperature semiclassical regime (9), with $\mu^{\text {qu }}$ much smaller than $\mu^{\text {se }}$. This can be understood in terms of smearing of the Coulomb blockade by the strong tunneling processes. Taking into account that at low temperatures the renormalized charging energy of the 
FSET, $E_{c}^{*}$, is 1 order of magnitude smaller than the bare charging energy $E_{c}$ when $\alpha_{\mathrm{M}}=10$ [17], we see that $\left(E_{c}^{*} / k_{B} T\right)^{2} \sim 0.01\left(E_{c} / k_{B} T\right)^{2}$, consistent with Eqs. (7) and (9) where $\mu^{\mathrm{qu}} / \mu^{\mathrm{se}} \sim 0.01$. The argument used here will fail at sufficiently low temperatures, where the TMR can hardly be described by a power law of $\left(E_{c} / k_{B} T\right)^{2}$. In such an extremely low temperature and strong tunneling regime, more investigations need to be done.

In conclusion, we have derived formulas for the resistance of the ferromagnetic single-electron transistor which are valid also in the regime when the junction resistances are smaller than the quantum resistance. In the strong tunneling regime we find that the magnetoresistance ratio is largely enhanced by the higher-order tunneling processes at low temperatures. The magnetoresistance ratio in this regime is much larger than the value predicted by including only sequential or cotunneling processes. We find that the TMR can at least be enhanced by a factor of 5 relative to the classical value at low temperatures. When the thermal energy is larger than the charging energy, the enhancement of the TMR is small, even when the junction resistances are much smaller than the quantum resistance.

The authors are grateful to G.E.W. Bauer, K.-A. Chao, J. Inou, A.N. Korotkov, Yu. V. Nazarov, and A. A. Odintsov for stimulating discussions and R. Egger for valuable support of the Monte Carlo simulations. X.H.W. would like to thank the Swedish TFR for financial support and A. B. acknowledges financial support from the EU TMR Research Network No. FMRX-CT960089 (DG12-MIHT).

*Also at: Philips Research Laboratories, Professor Holstlaan 4, 5656 AA Eindhoven, The Netherlands.

[1] M. Julliere, Phys. Lett. 54A, 225 (1975).

[2] J. S. Moodera, L. R. Kinder, T. M. Wong, and R. Meservey, Phys. Rev. Lett. 74, 3273 (1995); J. S. Moodera and L. R. Kinder, J. Appl. Phys. 79, 4724 (1996).
[3] P. M. Levy and S. F. Zhang, Curr. Opin. Solid State Mater. Sci. 4, 223 (1999).

[4] K. Ono, H. Shimada, and Y. J. Ootuka, J. Phys. Soc. Jpn. 66, 1261 (1997).

[5] L. F. Schelp, A. Fert, F. Fettar, P. Holody, S. F. Lee, J. L. Maurice, F. Petroff, and A. Vaures, Phys. Rev. B 56, R5747 (1997).

[6] S. Sankar, B. Dieny, and A. E. Berkowitz, J. Appl. Phys. 81, 5512 (1997).

[7] D. V. Averin and Yu. V. Nazarov, Phys. Rev. Lett. 65, 2446 (1990).

[8] D. V. Averin and K. K. Likharev, in Mesoscopic Phenomena in Solids, edited by B.L. Altshuler, P. A. Lee, and R. A. Webb (Elsevier, Amsterdam, 1991); G.-L. Ingold and Yu. V. Nazarov, in Single Charge Tunneling, edited by H. Grabert and M. H. Devoret, NATO Advanced Study Institutes, Ser. B, Vol. 294 (Plenum, New York, 1992).

[9] G. Schön and A. D. Zaikin, Phys. Rep. 198, 237 (1990), and references therein.

[10] X. H. Wang, Phys. Rev. B 55, 12868 (1997).

[11] J. Barnas and A. Fert, Phys. Rev. Lett. 80, 1058 (1998).

[12] A. Brataas, Yu. V. Nazarov, J. Inoue, and G. E. W. Bauer, Phys. Rev. B 59, 93 (1999); Eur. Phys. J. B 9, 421 (1999).

[13] A. Korotkov and V.I. Safarov, Phys. Rev. B 59, 89 (1999); Superlattices Microstruct. 25, 259 (1999).

[14] S. Takahashi and S. Maekawa, Phys. Rev. Lett. 80, 1758 (1998).

[15] H. Imamura, S. Takahashi, and S. Maekawa, Phys. Rev. B 59, 6017 (1999).

[16] M. Büttiker, Phys. Rev. B 36, 3548 (1987).

[17] X. H. Wang, R. Egger, and H. Grabert, Europhys. Lett. 38, 545 (1997), and references therein.

[18] G. D. Mahan, Many-Particle Physics (Plenum Press, New York, 1990), Chap. 3.

[19] S. Chakravarty and J. Rudnick, Phys. Rev. Lett. 75, 501 (1995); J.E. Hirsh, in Quantum Monte Carlo Methods in Equilibrium and Nonequilibrium Systems, edited by M. Suzuki (Springer, Berlin, 1987).

[20] P. Joyez, V. Bouchiat, D. Esteve, C. Urbina, and M. H. Devoret, Phys. Rev. Lett. 79, 1349 (1997). 